\title{
Numerical simulation of hemolysis: a comparison of Lagrangian and Eulerian modelling
}

\author{
S. Pirker ${ }^{1}$, H. Schima ${ }^{2} \&$ M. Stoiber ${ }^{2}$ \\ ${ }^{1}$ Johannes Kepler University, 4040 Linz, Austria \\ ${ }^{2}$ Medical University Vienna, 1090 Vienna, Austria
}

\begin{abstract}
In rotary blood pumps the degree of hemolysis is of crucial importance. Therefore, it would be desirable to account for flow induced hemolysis by means of numerical simulations in an early design stage.

In this paper two numerical simulation methods for the simulation of hemolysis are proposed. Hereby, a Lagrangian approach based on tracking distinct erythrocytes is compared to an Eulerian approach in which the erythrocytes are treated as a continuous species. Both models account for the local instantaneous stress as well as the stress exposure time.

The basic functionality and consistency of the above models is shown by simple test geometry. In a second step the Eulerian method is applied for the simulation of unsteady hemolysis in a rotary blood pump.

Keywords: CFD-simulation, rotary blood pump, hemolysis, Lagrangian modelling, Eulerian modelling, unsteady hemolysis.
\end{abstract}

\section{Introduction}

In recent years numerical flow simulations (CFD) have proven to be a valuable tool in designing artificial organs, i.e. rotary blood pumps (e.g. [1-3]. On one hand classical design criteria like pumping efficiency, pressure head and hydraulic losses can be addressed. One the other side flow induced hemolysis can be investigated.

In this paper two mathematical methods, namely the Lagrangian and the Eulerian approach, for the simulation of hemolysis are introduced and compared to each other. The principal functionality of the models is shown by a simple 
case study. In a second step the simulation of hemolysis during unsteady operation modes is addressed.

All modelling is realised within the framework of the commercial CFD package Fluent [4]. The simulations are performed on Dell workstations and typical calculation times range from some minutes for the first case study up to several days in the case of the semi-axial pump.

\section{Hemolysis - Lagrangian modelling}

Recent research work [5-13] agrees that hemolysis is at least influenced by both instantaneous shearing stresses as well as exposure time. Thus, the history of stress loading of an erythrocyte is said to influence the probability of cell damage. A common approach for the simulation of hemolysis is tracing the paths of distinct erythrocytes in a Lagrangian frame of reference.

In this Lagrangian model the path of an individual erythrocyte is determined by the local flow conditions. In this modelling concept any backward influence is neglected as well as interactions between erythrocytes. In the numerical model every trajectory represents a whole thread of distinct cells and is characterised by a particle mass flow rate, $\dot{m}_{p}$, as sketched in Figure 1.

a)

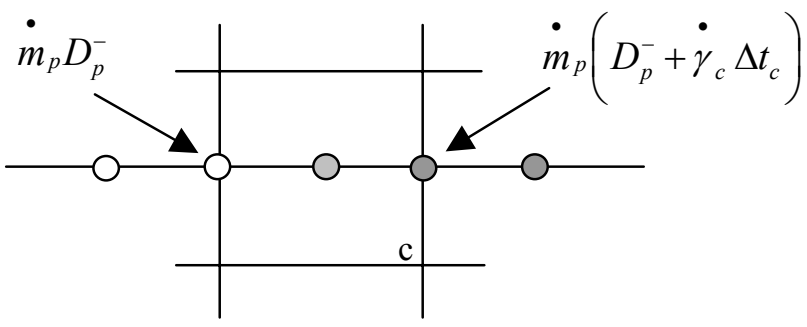

b)

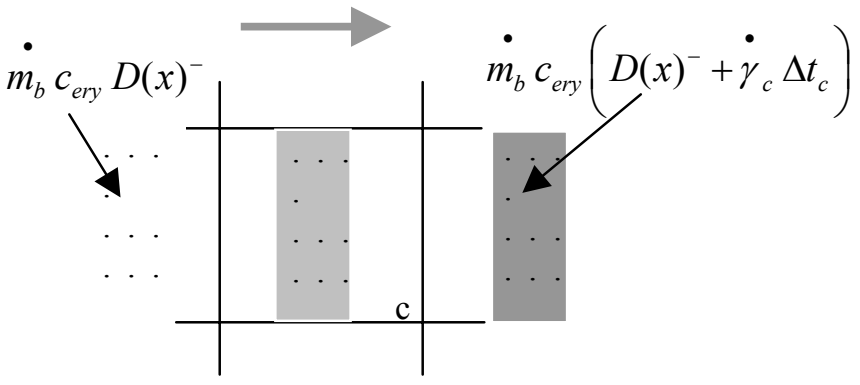

Figure 1: Integration of the blood damage potential in (a) Lagrangian and (b) Eulerian modelling.

During tracing the path of a particular erythrocyte the time-dependent stress loading can be monitored. In this model blood is assumed as a Newtonian fluid so that the instantaneous local stress $\sigma$ can by represented by the shearing rate $\dot{\gamma}$ and a constant viscosity $\mu$,

$$
\sigma=\mu \dot{\gamma}
$$


In three-dimensional flows the shearing rate $\dot{\gamma}$ is the square root of the second invariant of the rate of deformation tensor. If the stress exceeds a certain minimum activation level the model assumes that the cell will turn into an activated state described by a damage potential $D_{p}$.

If the stress loading continues the damage potential is increased by integrating the current strain rate over time. The simplest possibility to realise this integration on a computational grid is to sum up the product of the finite volume's strain rate, $\dot{\gamma}_{c}$, with the particle's residence time in that very computational cell, $\Delta t_{c}$. In Figure 1 the incoming particle of a distinct particle thread is described by an initial damage Potential $D_{p}^{-}$. During integration the damage potential rises to $D_{p}{ }^{-}+\sigma_{c} \Delta t_{c}$ until the thread's particles leave the very finite volume.

If this damage potential $D_{p}$ reaches a critical value the cell's membrane ruptures and the very erythrocyte will be irreversibly damaged. In this proposed model the evaluation of global hemolysis is not archived by counting the damaged erythrocytes in a huge set of introduced ones. Instead, the damaged cells are assumed to emit their hemoglobin into the blood's serum. In the mathematical model this is done by defining $\dot{m}_{p}$ as an adequate source term for the liquid phase's hemoglobin. Thereafter, the emitted hemoglobin is traced by an additional transport equation in an Eulerian frame of reference. As a consequence the hemolysis in the computational domain can be evaluated by simply looking at the calculated serum's free hemoglobin concentration at the domain's outlet.

\section{Hemolysis - Eulerian modelling}

If the distributed erythrocytes neither interfere with each others nor influence the main blood flow they can be treated as a passive continuous species. In this case an Eulerian equivalent of the above Lagrangian hemolysis model is possible. In this continuous model the flow rate of the erythrocytes is given by the blood flow rate, $\dot{m}_{b}$, times the mean erythrocyte concentration, $c_{\text {ery }}$.

To account for stress loading once again a damage potential is defined. In case of the Eulerian model this damage potential is given by a scalar field $D(\boldsymbol{x})$. This damage potential field can alternatively be interpreted as a concentration field of activated erythrocytes.

If in some finite volume the flow induced stress exceeds the activation level the damage potential $D(\boldsymbol{x})$ will be increased. This transformation is achieved by volumetric source terms. If several stress related finite volumes are passed successively the concentration of the activated erythrocytes will increase accordingly. In a similar way to above Lagrangian integration the activation state will be summed up until a rupture limit is reached. 


$$
\sum_{p} \dot{m}_{p} \dot{\gamma}_{c} \Delta t_{c}=\dot{m}_{b} c_{e r y} \dot{\gamma}_{c} \Delta t_{c}
$$

In the above equation the integration step of the Lagrangian model is compared to the Eulerian model. The left hand side describes the total change in the damage potential of all Lagrangian threads of erythrocytes passing a computational cell with a local strain rate $\dot{\gamma}_{c}$. In the right hand side the flux of the Eulerian damage potential field is increased accordingly.

If the cell membrane ruptures the activated erythrocyte species emits hemoglobin into the blood's serum like in the Lagrangian modelling. So an estimation of the degree of hemolysis is given once again by the concentration of the serum's free hemoglobin at the outlet of the computational domain.

\section{Case study 1 - simple stenosis configuration}

In the simple stenosis situation of Figure 2 blood passes two constrictions. In Figure $2 \mathrm{a}$ the corresponding velocity field is given. Based on the flow field the strain rate and therewith the stress distribution in the liquid is obtained as given in Figure 2b.

A distinct erythrocyte passing this geometry will experience several distinct stress maxima corresponding to the shearing rate caused by the two constrictions. A typical stress loading history is given in Figure 2c.

Based on above modelling concept most of the blood cells will be activated in the wall near region shortly before the first constriction. Some survive the first maximum stress but rupture shortly behind the second one. So applying this model the event of irreversible damage, say cell membrane's rupture, do not coincide with the moment of maximum stress loading. An extreme single stress event can lead to blood damage as well as a series of medium stresses experienced during a long exposure time.

In both modelling concepts, the estimation of hemolysis is based on an integration of stress and exposure time. In the Lagrangian approach this summation is done along erythrocytes' trajectories while in the Eulerian approach a continuous scalar field is integrated.

In Figure 3 the results of both hemolysis modelling approaches are given. Keeping in mind the uncertainties of the physiological mechanism of hemolysis both models deliver reasonable and consistent results. Obviously the basic modelling concept of integrating instantaneous stress times exposure time can be realised in a Lagrangian frame of reference as well as in an Eulerian one.

From a computational point of view the Eulerian model has some obvious advantages over the Lagrangian. The Lagrangian model requires several thousands of erythrocytes to be traced in order to receiving reliable and repeatable results. Therefore, this approach turns out to be very CPU intensive in technical applications. Another drawback for the Lagrangian model are numerical uncertainties in the integration of the distinct trajectories. During calculation erythrocytes might be trapped in boundary layers especially in narrow gap flows and adulterate the results. 
a)

b)
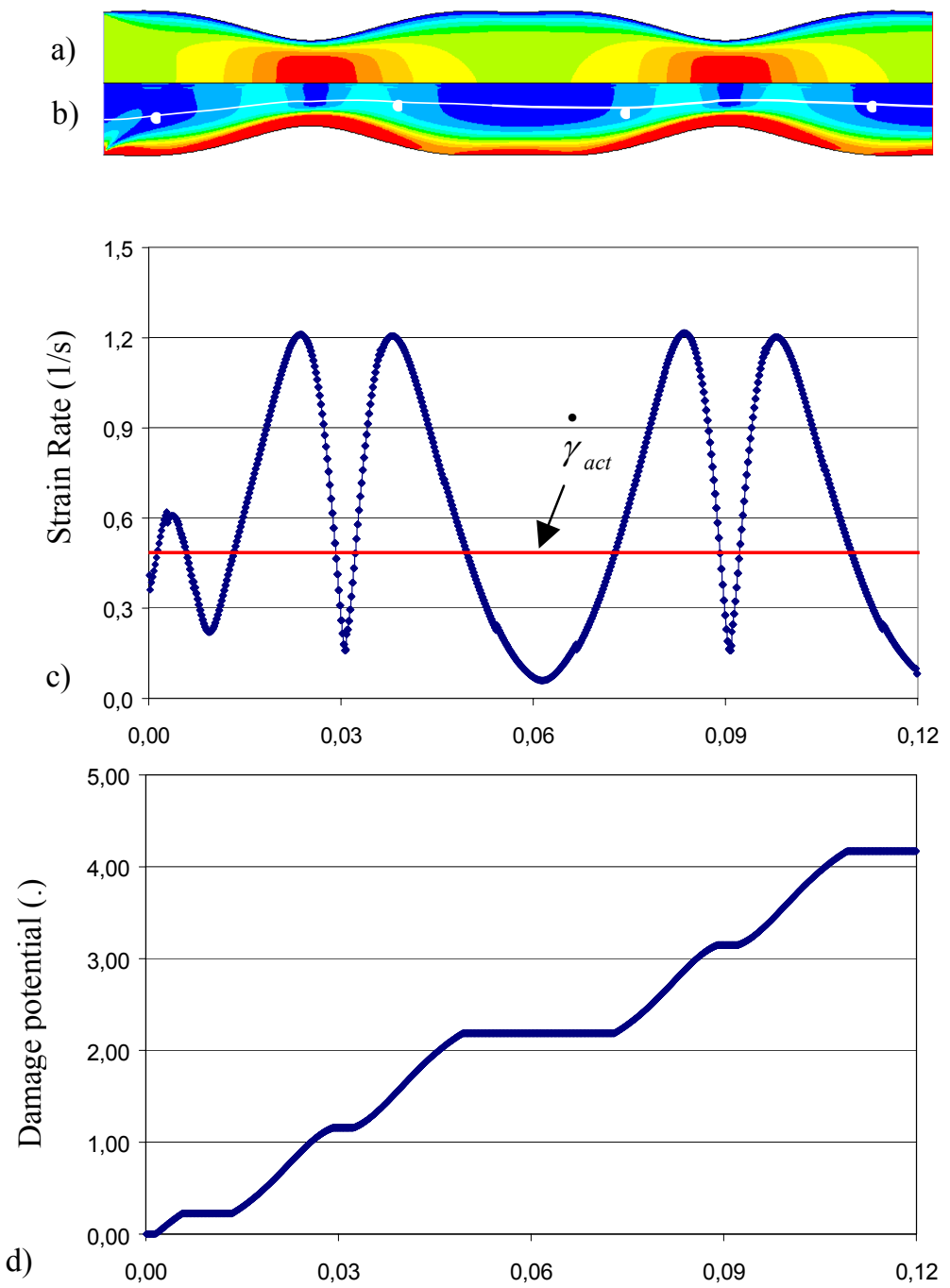

Length (m)

Figure 2: First test case with (a) velocity magnitude field $\left(\mathrm{v}_{\max }=0.3 \mathrm{~m} / \mathrm{s}\right)$ and (b) strain rate distribution $\left(\dot{\gamma}_{\max }=30001 / \mathrm{s}\right)$ as well as (c) strain rate history and (d) damage potential integration along a Lagrangian erythrocyte path. 
a)

b)
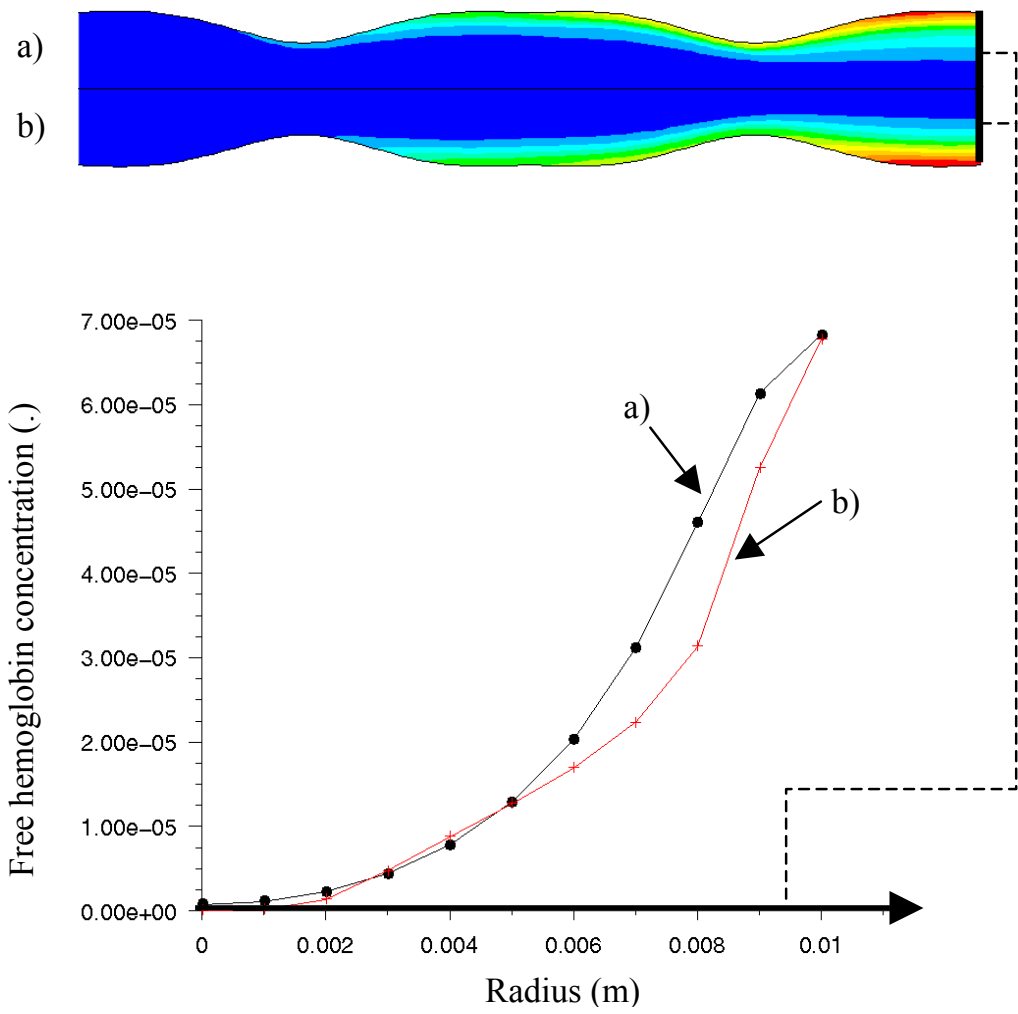

Figure 3: Concentration of the serum's free hemoglobin at the domain's outlet as result of (a) Lagrangian and (b) Eulerian modelling.

Another advantage of the Eulerian model is that unsteady simulations of hemolysis are possible without any modification of the mathematical model. An unsteady evaluation of hemolysis can easily be done by monitoring the timedependent concentration of the serum's free hemoglobin at the domain's outlet. Therefore, with help of this model the blood damage of a pulsing flow situation which e.g. occurs in implanted rotary blood pumps can be studied in detail.

\section{Case study 2 - rotary blood pump}

While the first case study concentrates on a comparison between the Lagrangian and the Eulerian hemolysis model the second case study emphasises on the possibility of unsteady hemolysis simulation by means of the Eulerian model.

The computational domain of the semi-axial pump considered in this case study starts at the input flange and ends at the pressure side ring volume of a semi-axial rotary blood pump. For sake of simplicity only one blade section of the impeller is considered. Blood is treated as a Newtonian fluid of constant properties $\left(\rho=1013 \mathrm{~kg} / \mathrm{m}^{3}, \mu=3.4 \mathrm{~kg} / \mathrm{m} . \mathrm{s}\right)$. At the pump inlet the flow velocity is fixed while on the ring outlet a constant static pressure is assumed. 


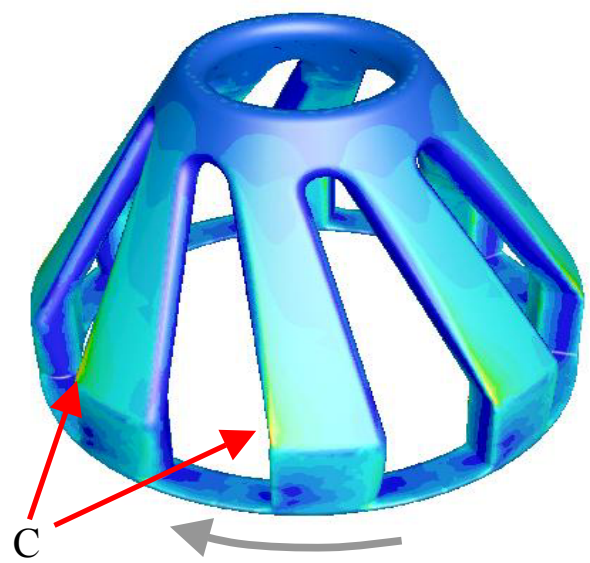

Figure 4: Wall shear stress plotted on the rotating impeller showing critical regions $(\mathrm{C})$ at the blade's leading edges.
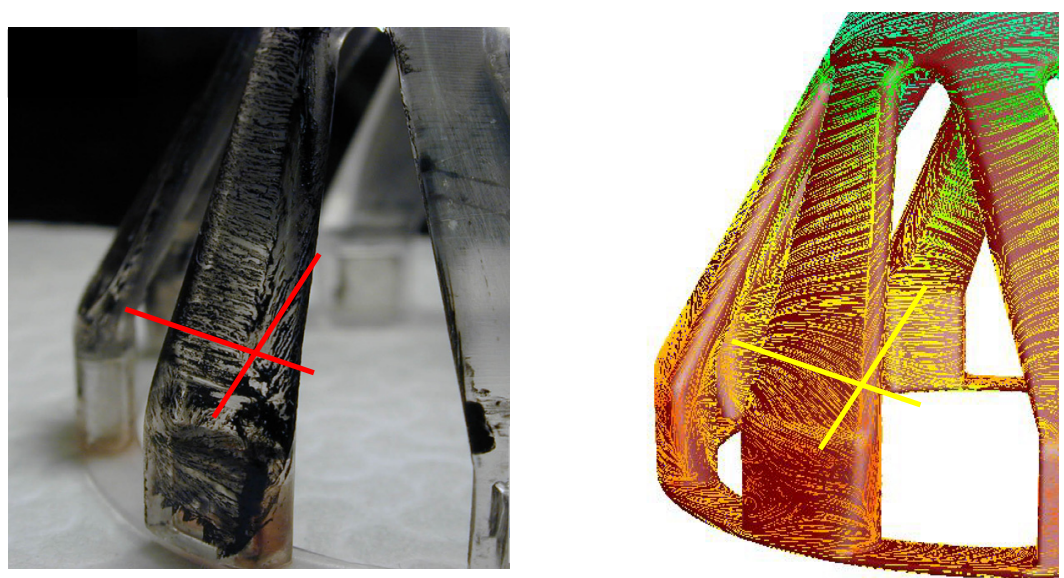

Figure 5: Colour abrasion experiments and simulated oil traces on the impeller. The orientation of the abrasion and the oil traces at the blade's trailing edge are given by the bold lines.

As a result of these simulations the time-dependent fully three-dimensional velocity and pressure distribution are obtained. Based on the flow situation the distribution of shearing stresses can be evaluated. In Figure 4 the wall shear stress is given on the impeller. The highest stresses can be observed at the leading edge of the impeller blades.

Because of the simplified computational domain global simulation results like pressure head and volumetric flow rate cannot directly compared to measurements. Therefore, a colour abrasion experiment was performed in order 
to depict local flow phenomena. In Figure 5 the orientation of the colour abrasion pattern agree well with the simulation of a corresponding wall oil flow.

The basic functionality of the Eulerian hemolysis model is shown during two unsteady pump operation modes. First, a stepwise increase in rotation speed is considered while secondly, the pump experiences a pulsing inflow.

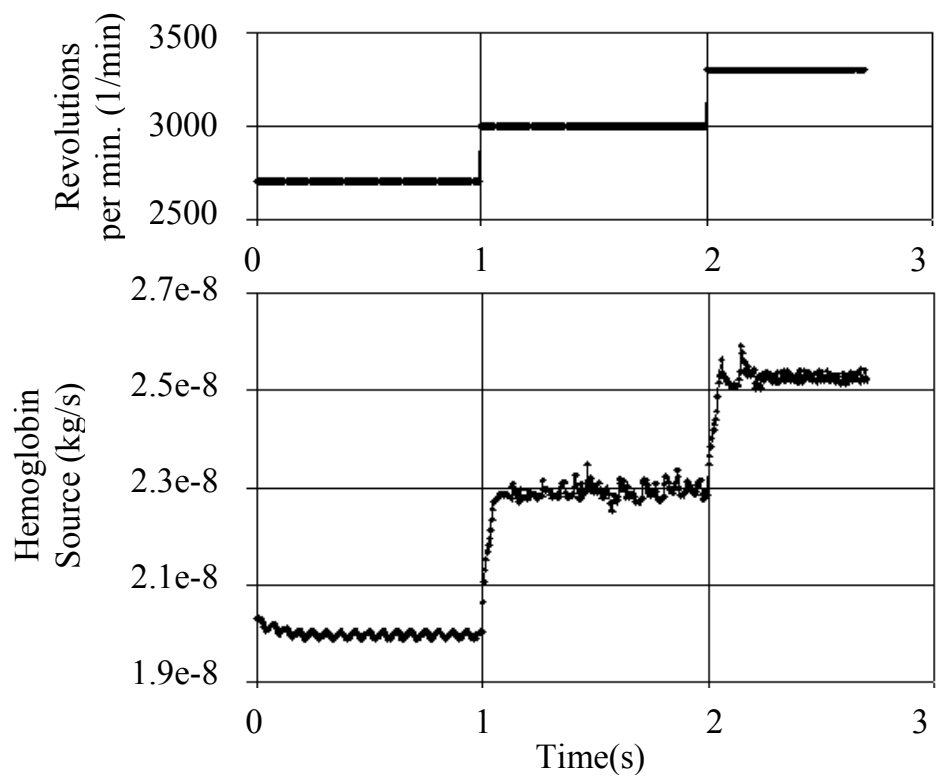

Figure 6: Simulated hemoglobin production in semi-axial LVAD pump during stepwise increase in rotational speed.

In Figure 6 the serum's hemoglobin production, thus the amount of hemolysis is plotted versus time. During the stepwise increase in the pump's rotation speed, the shear stress loading of the passing blood cells rises. As a consequence the global hemolysis of the pump is increased.

An important consideration is the behaviour of an implanted left ventricle assistant device (LVAD) in pulsing flow conditions. If a positive pressure pulse is defined at the suction inlet of the pump, the overall pump's pressure head decreases. At constant rotation speed the primary flow rate through the pump increases while any secondary leakage back flows calm down. From the viewpoint of hemolysis this operation mode is advantageous because both stress loading and exposure time are reduced. In Figure 7 the serum's hemoglobin production is plotted during an incoming positive pressure pulse. It clearly depicts that hemolysis is significantly reduced in that operation mode. 


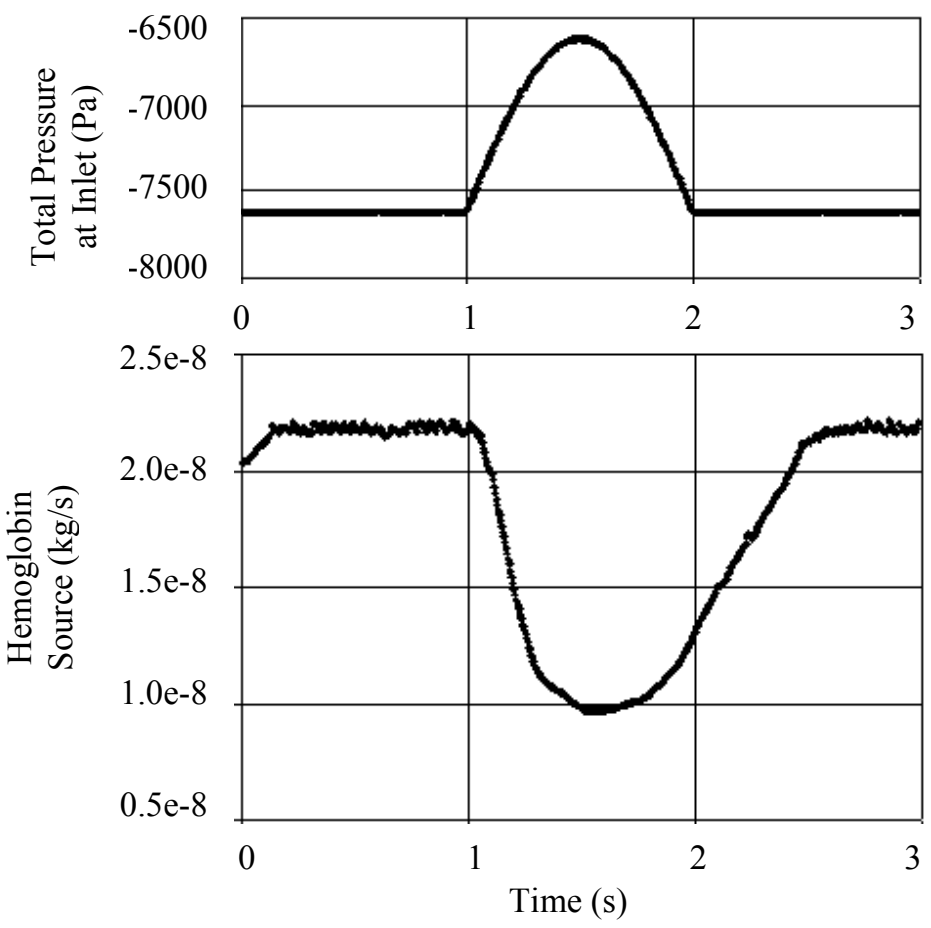

Figure 7: Simulated hemolysis in semi-axial LVAD pump during an inflow pressure pulse.

\section{Conclusion}

In this study two mathematical methods for the simulation of hemolysis are introduced. While in the Lagrangian model distinct erythrocytes are traced the Eulerian model treats the erythrocytes as a continuous species. Both models take into account the instantaneous stress as well as the exposure time.

In a first case study the two models are compared in a simple stenosis configuration. On principal both the Lagrangian and the Eulerian approach deliver comparable results. Nevertheless, the Eulerian model is by far more effective by means of computational costs.

In a second case study unsteady hemolysis phenomena in a semi-axial rotary blood pump are studied with the help of the Eulerian model. Pulsatile inflow conditions are addressed as well as changes in the pump's operation point.

Up to this point two mathematical approaches have been compared to each other and their principal functionality has been shown. They are by no means validated by real measurements what should be checked in near future. 


\section{References}

[1] Burgreen G.W. et al., Computational Fluid Dynamics as a Development Tool for Rotary Blood Pumps. Artificial Organs, 25(5), pp. 336-340, 2001.

[2] Bertram C.D., Qian Y. and Reizes J.A., Computational Fluid Dynamics Performance Prediction for the Hydrodynamic Bearings of a VentrAssist Rotary Blood Pump. Artificial Organs, 25(5), pp. 348-357, 2001.

[3] Chung M.K.H. et al., Impeller Behavior and Displacement of the VentrAssist Implantable Rotary Blood Pump. Artificial Organs, 28(3), pp. 287-297, 2004.

[4] Fluent, Fluent 6.1 User Guide. Fluent Inc., Lebanon, USA, 2003.

[5] Paul R. et al., Shear Stress Related Blood Damage in Laminar Couette Flow. Artificial Organs, 27(6), pp. 517-529, 2003.

[6] Yeleswarapu K.K., A Mathematical Model for Shear-Induced Hemolysis. Artificial Organs, 19(7), pp. 576-582, 1995.

[7] Bludszuweit C., Model for a General Mechanical Blood Damage Prediction. Artificial Organs, 19(7), pp. 583-589, 1995.

[8] Bludszuweit C., Three-Dimensional Numerical Prediction of Stress Loading of Blood Particles in a Centrifugal Pump. Artificial Organs, 19(7), pp. 583-589, 1995.

[9] Arora D. et al., A Tensor Based Measure for Estimating Blood Damage. Artificial Organs, 28(11), pp. 1002-1015, 2004.

[10] Apel J. et al., Assessment of Hemolysis Related Quantities in Microaxial Blood Pumps by Computational Fluid Dynamics. Artificial Organs, 25(5), pp. 341-347, 2001.

[11] Garon A. and Farina M-I., Fast Three-dimensional Numerical Hemolysis Approximation. Artificial Organs, 28(11), pp. 1016-1025, 2004.

[12] Goubergrits L. and Affelt K., Numerical Estimation of Blood Damage in Artificial Organs. Artificial Organs, 28(11), pp. 499-507, 2004.

[13] Grigioni M. et al., The Power-law Mathematical Model for Blood Damage Prediction. Artificial Organs, 28(5), pp. 467-475, 2004. 